\title{
Robust Possibilistic Optimization with Copula Function
}

\author{
Romain Guillaume \\ Université de Toulouse-IRIT \\ Toulouse, France \\ romain.guillaume@irit.fr
}

\author{
Adam Kasperski \\ Wroctaw University of Science \\ and Technology \\ Wrocław, Poland \\ adam.kasperski@pwr.edu.pl
}

\author{
Paweł Zieliński \\ Wroctaw University of Science \\ and Technology \\ Wrocław, Poland \\ pawel.zielinski@pwr.edu.pl
}

\begin{abstract}
This paper deals with a linear optimization problem with uncertain objective function coefficients modeled by possibility distributions. The fuzzy robust optimization framework is applied to compute a solution. Namely, the necessity degree that the objective value is lower than a given threshold is maximized. The aim of this paper is to take the knowledge on dependencies between the objective coefficients into account by means of a family of copula functions. It is shown that this new approach limits the conservatism of fuzzy robust optimization, better evaluates possibility distributions for the values of the objective function and do not increase the complexity of the problem.
\end{abstract}

Index Terms-robust optimization; possibility theory; random fuzzy set

\section{INTRODUCTION}

In this paper we study the following optimization problem with uncertain objective function coefficients:

$$
\begin{array}{ll}
\min & \tilde{\boldsymbol{c}}^{T} \boldsymbol{x}, \\
\mathrm{s.t.} & \boldsymbol{a}_{i}^{T} \boldsymbol{x} \geq b_{i} \quad i \in[\mathrm{m}], \\
& \boldsymbol{x} \geq \mathbf{0} .
\end{array}
$$

In formulation (1), $x$ is $n$-vector of nonnegative decision variables, $\tilde{\boldsymbol{c}}$ is $n$-vector of uncertain cost function coefficients, $\boldsymbol{a}_{i}=\left(a_{i 1}, \ldots, a_{i n}\right)$ is $n$-vector of deterministic coefficients of the $i$ th constraint, $i \in[m]([m]$ denotes the set $\{1, \ldots, m\})$. We assume that the right-hand side $m$-vector $b$ is precisely known.

A method of solving (1) depends on the information available on the objective function coefficients. This information can be imprecise. One of the traditional frameworks to handle imprecision is the possibility theory [1]. Let $\Omega$ be a set of elementary events. The possibility theory enables to encode a family of probability distributions in $\Omega$ thought a possibility distribution $\pi, \pi: \Omega \rightarrow[0,1]$, which assigns to each elementary event $\omega \in \Omega$ a possibility degree $\pi(\omega)$ (see, e.g., [2]). The value $\pi(\omega)=1$ means that $\omega$ is totally possible and $\pi(\omega)=0$ means that $\omega$ is impossible. Any subset $A$ of $\Omega$ is called an event. The possibility distribution $\pi$ induces two dual measures defined on the set of subsets of $\Omega$, namely the possibility and the necessity ones, denoted by $\Pi$ and $\mathrm{N}$, respectively, and encodes a family of probability distributions

Romain Guillaume was partially supported by the AI Interdisciplinary Institute ANITI Grant no. ANR-19-PI3A-0004. in $\Omega$ such $\mathrm{N}(A) \leq \operatorname{Pr}(A) \leq \Pi(A)$ for every $A \subseteq \Omega$, where $\Pi(A)$ and $\mathrm{N}(A)$ are the possibility and necessity degrees of $A$, respectively. Thus $\Pi(A)$ and $\mathrm{N}(A)$ are upper and lower bounds on the probability of $A$ (see, e.g [2]).

Let $\mathcal{U} \subseteq \mathbb{R}^{n}$ be uncertainty set containing possible realizations (scenarios) of uncertain objective function coefficients $\tilde{\boldsymbol{c}}$. It is assumed that a possibility distribution $\pi_{\tilde{\boldsymbol{c}}}, \pi_{\tilde{\boldsymbol{c}}}: \mathcal{U} \rightarrow[0,1]$, is specified in $\mathcal{U}$. It induces a possibility distribution $\pi_{\tilde{c}^{T} \boldsymbol{x}}$, $\pi_{\tilde{\boldsymbol{c}}^{T} \boldsymbol{x}}: \mathbb{R} \rightarrow[0,1]$, of uncertain objective function values $\tilde{\boldsymbol{c}}^{T} \boldsymbol{x}$ for a given feasible solution $\boldsymbol{x}$. Several approaches to handle uncertainty in (1) have been proposed in the existing literature. One of the most common approaches is to provide a goal (budget) $g$ and compute a solution whose cost is lower than $g$ under possible realizations of $\tilde{\boldsymbol{c}}$ - both in robust (possibilistic) and stochastic settings (see, e.g., [3]-[5]). In robust possibilistic optimization the objective is to maximize the degree of certainty that a solution cost is lower than $g$, namely the necessity based criterion: $\mathrm{N}\left(\tilde{\boldsymbol{c}}^{T} \boldsymbol{x} \leq g\right)=1-\sup \left\{\pi_{\tilde{\boldsymbol{c}}^{T} \boldsymbol{x}}(u)\right.$ : $u>g\}$ is maximized (see, e.g. [6]). Thus a robust possibilistic counterpart of problem (1) can be formulated as follows:

$$
\begin{array}{ll}
\max & \mathrm{N}\left(\tilde{\boldsymbol{c}}^{T} \boldsymbol{x} \leq g\right) \\
\text { s.t. } & \boldsymbol{a}_{i}^{T} \boldsymbol{x} \geq b_{i} \\
& \boldsymbol{x} \geq \mathbf{0}
\end{array} \quad i \in[m],
$$

In our model the uncertainty of cost coefficients $\tilde{c}_{j}, j \in[n]$, is represented by triangular fuzzy intervals $\tilde{c}_{j}=\left(\widehat{c}_{j}-\right.$ $\Delta_{j}^{-}, \hat{c}_{j}, \hat{c}_{j}+\Delta_{j}^{+}$), where $\hat{c}_{j}$ is the nominal value (the most possible value of $\tilde{c}_{j}$ ) and $\Delta_{j}^{-}$and $\Delta_{j}^{+}$are the maximal deviations of values of $\tilde{c}_{j}$ from $\hat{c}_{j}$. The membership function $\pi_{\tilde{c}_{j}}$ of fuzzy interval $\tilde{c}_{j}$ is interpreted as a possibility distribution for $\tilde{c}_{j}$, obviously $\pi_{\tilde{c}_{j}}\left(\hat{c}_{j}\right)=1$.

A multivariate possibility distribution $\pi_{\tilde{c}}$ in uncertainty set $\mathcal{U}$ can be built from marginal possibility distributions for cost coefficients $\pi_{\tilde{c}_{j}}, j \in[n]$. Typically the dependencies among the uncertain cost coefficients $\tilde{c}_{j}$ are ignored and usually a multivariate distribution $\pi_{\tilde{c}}$ with the minimum aggregator is chosen as a precaution (see, e.g., [3], [7], [8]). In this paper, we show how to build a more precise multivariate possibility distribution $\pi_{\tilde{c}}$ by taking into account the available knowledge on both positive and negative dependencies between $\tilde{c}_{j}, j \in$ $[n]$. In order to do this we adopt an approach used in the 
probabilistic context, which is based on copulas (see, e.g., [9], [10]) and apply it in the possibilistic one.

\section{Copulas in Nutshell}

In this section we briefly recall the notion of a copula and some properties, following [10], [11], that are important for its understanding and interpretation. In the probabilistic setting a copula $C:[0,1]^{n} \rightarrow[0,1]$ is a cumulative distribution function with marginals uniformly distributed on $[0,1]$. In the analytical one a copula function can characterized as follows.

Theorem 1: Let $C(\boldsymbol{u})=C\left(u_{1}, \ldots, u_{n}\right)$. Function $C$ is a copula if and only if the following conditions hold:

(i) $C\left(1, \ldots, 1, u_{j}, 1, \ldots, 1\right)=u_{j}$ for every $j \in[n]$, where $u_{j} \in[0,1]$,

(ii) $C\left(u_{1}, \ldots, u_{n}\right)$ is nondecreasing in each component $u_{j}$,

(iii) for each hyperrectangle $H \subseteq[0,1]^{n}$ the $C$-volume of $H$ is nonnegative, i.e. $\int_{H} \mathrm{~d} C(\boldsymbol{u}) \geq 0$.

Let $\mathcal{C}_{n}$ denote the set of $n$ - dimensional copulas. Theorem 1 , implies $\mathcal{C}_{n}$ is a convex set. Important examples of copulas are: the independence copula $\prod_{i \in[n]} u_{i}$, positive dependence copula (or comonotonicity copula) $\min _{i \in[n],}\left\{u_{i}\right\}$ and negative dependence copula (or countermonotonicity copula) $W\left(u_{1}, u_{2}\right)=\max \left\{u_{1}+u_{2}-1,0\right\}$. The following theorem gives upper and lower bounds in $\mathcal{C}_{n}$.

Theorem 2 (Fréchet-Hoeffding bounds): For any copula $C \in$ $\mathcal{C}_{n}$ and any $\boldsymbol{u} \in[0,1]^{n}$ the following inequalities hold:

$$
W(\boldsymbol{u}) \leq C(\boldsymbol{u}) \leq M(\boldsymbol{u}),
$$

where $W(\boldsymbol{u})=\max \left\{\sum_{j \in[n]} u_{j}-n+1,0\right\}$ and $M(\boldsymbol{u})=$ $\min _{j \in[n]}\left\{u_{j}\right\}$.

It is worth pointing out that $W$ is not a copula for $n \geq 3$.

We now recall the fundamental theorem of copulas (Sklar's Theorem) that provides the theoretical foundation for their applications. It elucidates the role that copula plays in the relationship between multivariate distribution functions and their univariate margins.

Theorem 3 (Sklar): If $F_{i}\left(c_{j}\right)=\operatorname{Pr}\left(\mathbf{C}_{j} \leq c_{j}\right), j \in[n]$, are univariate distribution functions, where $\mathbf{C}_{j}, j \in[n]$, are random variables, and $C \in \mathcal{C}_{n}$, then a joint distribution function $F:[-\infty,+\infty]^{n} \rightarrow[0,1]$ defined as follows:

$$
F(\boldsymbol{c})=C\left(F_{1}\left(c_{1}\right), \ldots, F_{n}\left(c_{n}\right)\right)=\operatorname{Pr}\left(\bigcap_{j \in[n]}\left\{\mathbf{C}_{j} \leq c_{j}\right\}\right)
$$

is an $n$-dimensional distribution function with margins $F_{j}, j \in$ $[n]$.

One can also give a version of Theorem 3 formulated in terms of survival functions $\bar{F}_{i}\left(c_{j}\right)=\operatorname{Pr}\left(\mathbf{C}_{j}>c_{j}\right), j \in[n]$, that has applications in risk models. Thus

$$
\bar{F}(\boldsymbol{c})=\breve{C}\left(\bar{F}_{1}\left(c_{1}\right), \ldots, \bar{F}_{n}\left(c_{n}\right)\right)=\operatorname{Pr}\left(\bigcap_{j \in[n]}\left\{\mathbf{C}_{j}>c_{j}\right\}\right)
$$

for some copula $C \in \mathcal{C}_{n}$ called the survival copula.

\section{ROBUST POSSIBILISTIC OPTIMIZATION USING COPULAS}

Suppose that we are given a possibility distribution $\pi_{\tilde{\boldsymbol{c}}}$ : $\mathbb{R}^{n} \rightarrow[0,1]$ of an uncertain vector $\tilde{\boldsymbol{c}}$ (an uncertain cost vector). Without loss of generality we can assume that $\pi_{\tilde{\boldsymbol{c}}}$ is a possibility distribution in uncertainty set $\mathcal{U} \subseteq \mathbb{R}^{n}$. Then using the possibility theory (see, e.g., [1]) one can build possibility and necessity measures, $\Pi$ and $N$. Therefore, the degree of possibility and the degree of necessity of event " $\tilde{c} \in A$ ", where $A$ is a subset of $\mathcal{U}$, are as follows:

$$
\begin{aligned}
& \Pi(\tilde{\boldsymbol{c}} \in A)=\sup _{\boldsymbol{u} \in A} \pi_{\tilde{\boldsymbol{c}}}(\boldsymbol{u}), \\
& \mathrm{N}(\tilde{\boldsymbol{c}} \in A)=1-\Pi(\tilde{\boldsymbol{c}} \notin A)=1-\sup _{\boldsymbol{u} \notin A} \pi_{\tilde{\boldsymbol{c}}}(\boldsymbol{u}) .
\end{aligned}
$$

On the other hand if $\Pi\left(\tilde{c}_{j} \in A_{j}\right), j \in[n]$, are given, where $A_{j}$ is the projection of $A$ on the $j$ th dimension, then $\Pi(\tilde{c} \in A)$ and $\mathrm{N}(\tilde{\boldsymbol{c}} \in A)$ can be built using the copula $M$, which is a common approach, namely

$$
\begin{aligned}
& \Pi(\tilde{\boldsymbol{c}} \in A)=\Pi\left(\bigcap_{j \in[n]}\left\{\tilde{c}_{j} \in A_{j}\right\}\right)=\min _{j \in[n]} \Pi\left(\tilde{c}_{j} \in A_{j}\right), \\
& \mathrm{N}(\tilde{\boldsymbol{c}} \in A)=1-\min _{j \in[n]} \Pi\left(\tilde{c}_{j} \notin A_{j}\right) .
\end{aligned}
$$

In fact, in context of ignorance on copula/survival copula functions we want to guarantee that $\Pi$ (resp. $\mathrm{N}$ ) is an upper bound (resp. lower bound) over all possibles copula/survival functions hence $n$-dimensional distributions. This is very extreme point of view since it means that in case of possibility (resp. necessity) all good (resp. bad) things will occur almost surely simultaneously. To moderate this point of view, we propose to use knowledge on copula function and marginal possibility distribution to evaluate the possibility and the necessity measure for a $n$-dimensional distribution. Accordingly, suppose that univariate possibility distributions $\pi_{\tilde{c}_{j}}$ of $\tilde{c}_{j}, j \in[n]$ are given. They induce univariate functions: $\Pi_{j}\left(\tilde{c}_{j} \leq c_{j}\right)$ and $\mathrm{N}_{j}\left(\tilde{c} \leq c_{j}\right)$ as follows:

$$
\begin{aligned}
\Pi_{j}\left(\tilde{c}_{j} \leq c_{j}\right) & =\sup _{u: u \leq c_{j}} \pi_{\tilde{c}_{j}}(u), \\
\mathrm{N}_{j}\left(\tilde{c} \leq c_{j}\right) & =1-\Pi_{j}\left(\tilde{c}_{j}>c_{j}\right)=1-\sup _{u: u>c_{j}} \pi_{\tilde{c}_{j}}(u) .
\end{aligned}
$$

In $n$-dimensional case we have a similar relation, namely

$$
\begin{aligned}
\mathrm{N}(\tilde{\boldsymbol{c}} \leq \boldsymbol{c})=\mathrm{N}\left(\bigcap_{j \in[n]}\left\{\tilde{c}_{j} \leq c_{j}\right\}\right) & =1-\Pi(\tilde{\boldsymbol{c}}>\boldsymbol{c}) \\
& =1-\Pi\left(\bigcap_{j \in[n]}\left\{\tilde{c}_{j}>c_{j}\right\}\right) .
\end{aligned}
$$

For simplicity of notation, we write $\Pi_{j}\left(c_{j}\right)$ (resp. $\bar{\Pi}_{j}\left(c_{j}\right)$ instead of $\Pi_{j}\left(\tilde{c}_{j} \leq c_{j}\right)$ (resp. $\Pi_{j}\left(\tilde{c}_{j}>c_{j}\right)$ ) and similarly for the $n$-dimensional case, we write $\Pi(\boldsymbol{c})$ (resp. $\bar{\Pi}(\boldsymbol{c})$ ) instead of $\Pi(\tilde{\boldsymbol{c}} \leq \boldsymbol{c})$ (resp. $\Pi(\tilde{\boldsymbol{c}}>\boldsymbol{c}))$. Furthermore, from now on, we call $\Pi_{j}\left(c_{j}\right)$ (resp. $\bar{\Pi}_{j}\left(c_{j}\right)$ and $\Pi(\boldsymbol{c})$ (resp. $\bar{\Pi}(\boldsymbol{c})$ ) possibility distribution functions (resp. possibility survival functions). 
By Theorem 3, we can now construct a possibility distribution function $\Pi(\boldsymbol{c})$ and a possibility survival function $\bar{\Pi}(\boldsymbol{c})$ :

$$
\begin{aligned}
& \Pi(\boldsymbol{c})=C\left(\Pi_{1}\left(c_{1}\right), \ldots, \Pi_{n}\left(c_{n}\right)\right), \\
& \bar{\Pi}(\boldsymbol{c})=\breve{C}\left(\bar{\Pi}_{1}\left(c_{1}\right), \ldots, \bar{\Pi}_{n}\left(c_{n}\right)\right),
\end{aligned}
$$

where $C$ and $\breve{C}$ are any copulas from $\mathcal{C}_{n}$. Note that (9) and (10) do not precisely define an $n$-dimensional possibility distribution function (resp. possibility survival function) but they define a set of possibility distribution functions (resp. possibility survival functions). Thus let $\mathcal{P}_{(C, \breve{C})}$ be the set of $n$-dimensional possibility distributions $\pi_{\tilde{c}}$ that induce $\Pi(c)$ and $\bar{\Pi}(\boldsymbol{c})$ satisfying, respectively, equations (9) and (10). We now claim that $\mathcal{P}_{(C, \breve{C})}$ is not empty. The $\lambda$-cuts defined as follows:

$$
\begin{array}{r}
\left\{\boldsymbol{c} \in \mathbb{R}^{n}: C\left(\Pi_{1}\left(c_{1}\right), \ldots, \Pi_{n}\left(c_{n}\right)\right) \geq \lambda,\right. \\
\left.\breve{C}\left(\bar{\Pi}_{1}\left(c_{1}\right), \ldots, \bar{\Pi}_{n}\left(c_{n}\right)\right) \geq \lambda\right\}
\end{array}
$$

satisfy (9) and (10). Now we need to show that a distribution built from these $\lambda$-cuts is a possibility distribution, i.e. the $\lambda$-cuts must satisfy the inclusion property and there exists $c \in \mathcal{U}$ whose possibility degree is equal to 1 .

Proposition 1: The $\lambda$-cuts defined by (11) satisfy the inclusion property.

Proof: See Appendix.

Proposition 2: The $\lambda$-cut for $\lambda=1$ is not empty.

Proof: See Appendix.

We thus get the following lemma

Lemma 1: $\mathcal{P}_{(C, \breve{C})}$ is not empty.

We are now ready to give the possibility and necessity degrees of event " $\tilde{c}^{T} \boldsymbol{x} \leq g$ " for a given $\boldsymbol{x}$.

Proposition 3: Let $x$ be a feasible solution to (1). For any $\pi_{\tilde{\boldsymbol{c}}} \in \mathcal{P}_{(C, \breve{C})}$, the possibility degree of event " $\tilde{\boldsymbol{c}}^{T} \boldsymbol{x} \leq g$ " is

$$
\Pi\left(\tilde{\boldsymbol{c}}^{T} \boldsymbol{x} \leq g\right)=\sup _{\boldsymbol{c}: \boldsymbol{c}^{T} \boldsymbol{x} \leq g} C\left(\Pi_{1}\left(c_{1}\right), \ldots, \Pi_{n}\left(c_{n}\right)\right) .
$$

Proof: See Appendix.

Proposition 4: Let $\boldsymbol{x}$ be a feasible solution to (1). For any $\pi_{\tilde{\boldsymbol{c}}} \in \mathcal{P}_{(C, \breve{C})}$, the necessity degree of event " $\tilde{\boldsymbol{c}}^{T} \boldsymbol{x} \leq g$ " is

$$
\mathrm{N}\left(\tilde{\boldsymbol{c}}^{T} \boldsymbol{x} \leq g\right)=1-\sup _{\boldsymbol{c}: \boldsymbol{c}^{T} \boldsymbol{x}>g} \breve{C}\left(\bar{\Pi}_{1}\left(c_{1}\right), \ldots, \bar{\Pi}_{n}\left(c_{n}\right)\right) .
$$

Proof: See Appendix.

In this paper, we propose to use a parametric copula that takes into account the knowledge about positive/negative dependencies among uncertain cost coefficients. The copula can be defined as follows:

Definition 1: $H_{\alpha}(\boldsymbol{u})=\alpha W(\boldsymbol{u})+(1-\alpha) M(\boldsymbol{u})$ for every $\alpha \in[0,1]$.

The parameter $\alpha$ models the belief that the copula is the most negative dependence copula for a given $W(\boldsymbol{u})$, while $1-\alpha$ is the belief that the copula is the most positive dependence copula for a given $M(\boldsymbol{u})$. Hence, if $\alpha=0$ we come back to the classical one which considers positives dependencies among uncertain cost function coefficients. The cost function coefficients increase simultaneously. If $\alpha=1$, we have negatives dependencies among uncertain cost function coefficients. Thus when one cost function coefficient increases the others decrease. Accordingly, parameter $\alpha$ is the degree that dependencies are positive.

Now we show a parametric function $H_{\alpha}$ which can be used to compute the necessity degree of event " $\tilde{c}^{T} \boldsymbol{x} \leq g$ " for a given $x$.

Proposition 5: For $n=2 H_{\alpha}(\boldsymbol{u})=\alpha W(\boldsymbol{u})+(1-\alpha) M(\boldsymbol{u})$ is a copula function for every $\alpha \in[0,1]$.

Proof: See Appendix.

Proposition 6: For any $n \geq 3$, any $\boldsymbol{u} \in[0,1]^{n}$ and any $\alpha \in[0,1]$, there exists a copula $C \in \mathcal{C}_{n}$ such that $C(\boldsymbol{u})=$ $H_{\alpha}(\boldsymbol{u})=\alpha W(\boldsymbol{u})+(1-\alpha) M(\boldsymbol{u})$.

Propositions 5, and 6 and (10) yield a necessity function:

$$
\mathrm{N}_{\alpha}(\tilde{\boldsymbol{c}} \leq \boldsymbol{c})=\mathrm{N}(\boldsymbol{c})=1-H_{\alpha}\left(\bar{\Pi}_{1}\left(c_{1}\right), \ldots, \bar{\Pi}_{n}\left(c_{n}\right)\right)
$$

and the necessity degree of event " $\tilde{c}^{T} \boldsymbol{x} \leq g$ " for a given $\boldsymbol{x}$ :

$$
\mathrm{N}_{\alpha}\left(\tilde{\boldsymbol{c}}^{T} \boldsymbol{x} \leq g\right)=1-\sup _{\boldsymbol{c}: \boldsymbol{c}^{T} \boldsymbol{x}>g} H_{\alpha}\left(\bar{\Pi}_{1}\left(c_{1}\right), \ldots, \bar{\Pi}_{n}\left(c_{n}\right)\right) .
$$

Proposition 7: Let $\epsilon \in[0,1]$. Then $\mathrm{N}_{\alpha}\left(\tilde{\boldsymbol{c}}^{T} \boldsymbol{x} \leq g\right)>1-\epsilon$ iff for each $c$ such that $H_{\alpha}\left(\bar{\Pi}_{1}\left(c_{1}\right), \ldots, \bar{\Pi}_{n}\left(c_{n}\right)\right)>\epsilon$, the constraint $\boldsymbol{c}^{T} \boldsymbol{x} \leq g$ is satisfied.

Proof: See Appendix.

Proposition 7 now shows that the problem (2) with $\mathrm{N}_{\alpha}\left(\tilde{\boldsymbol{c}}^{T} \boldsymbol{x} \leq\right.$ g) can be reformulated:

$$
\begin{array}{rll}
\min & \epsilon \\
\text { s.t. } & \max \boldsymbol{c}_{\alpha}\left(\bar{\Pi}_{1}\left(c_{1}\right), \ldots, \bar{\Pi}_{n}\left(c_{n}\right)\right) \geq \epsilon & \\
& \boldsymbol{a}_{i}^{T} \boldsymbol{x} \geq b_{i} \leq g, & \\
& \boldsymbol{x} \geq \mathbf{0}, \epsilon \in[0,1] . &
\end{array}
$$

Note that the problem (13) contains a maximization problem $\max _{\boldsymbol{c}: H_{\alpha}}\left(\bar{\Pi}_{1}\left(c_{1}\right), \ldots, \bar{\Pi}_{n}\left(c_{n}\right)\right) \geq \epsilon \boldsymbol{c}^{T} \boldsymbol{x}$ called adversarial problem. For simplicity, we denote by $\mathcal{H}_{\epsilon}^{\alpha}$ the set $\{\boldsymbol{c}$ : $\left.H_{\alpha}\left(\bar{\Pi}_{1}\left(c_{1}\right), \ldots, \bar{\Pi}_{n}\left(c_{n}\right)\right) \geq \epsilon\right\}$. Now the adversarial problem has following form:

$$
\text { ADV: } \max _{\boldsymbol{c} \in \mathcal{H}_{\epsilon}^{\alpha}} \boldsymbol{c}^{T} \boldsymbol{x}
$$

where $x$ is a given feasible solution. In the next section we show how to solve the adversarial problem.

\section{Resolution of Adversarial Problem}

The adversarial problem (14) is a maximization of $\boldsymbol{c}^{T} \boldsymbol{x}$ over the set $\mathcal{H}_{\epsilon}^{\alpha}$. Thus a structure of the feasible solution set $\mathcal{H}_{\epsilon}^{\alpha}$ is essential for solving the problem. Let us study the set $\mathcal{H}_{\epsilon}^{\alpha}$.

We first look at the extreme cases, namely where $\alpha=0$ and $\alpha=1$. Let us define two sets:

$$
\mathcal{M}^{\epsilon}= \begin{cases}\left\{x_{j \in[n]}\left(-\infty, \hat{c}_{j}+(1-\epsilon) \Delta_{j}^{+}\right]\right\} & \text {if } \epsilon \in(0,1], \\ \mathbb{R}^{n} & \text { if } \epsilon=0,\end{cases}
$$

and

$$
\mathcal{W}^{\epsilon}= \begin{cases}\left\{\times_{j \in[n]}\left(-\infty, \widehat{c}_{j}+\left(1-u_{j}\right) \Delta_{j}^{+}\right]:\right. & \\ \sum_{j \in[n]} u_{j}-n+1=\epsilon, \boldsymbol{u} \in[0,1]^{n} & \text { if } \epsilon \in(0,1], \\ \mathbb{R}^{n} & \text { if } \epsilon=0 .\end{cases}
$$

From definition of $H_{\alpha}$ we get $\mathcal{H}_{\epsilon}^{0}=\mathcal{M}^{\epsilon}$ and $\mathcal{H}_{\epsilon}^{1}=\mathcal{W}^{\epsilon}$. 
Proposition 8: The maximum and minimum values of $W$ and $M$ are:

$$
\begin{aligned}
& W_{\alpha, \epsilon}^{M}=\min \left\{1, \frac{\epsilon}{\alpha}\right\}, M_{\alpha, \epsilon}^{M}=\min \left\{1, \frac{\epsilon}{1-\alpha}\right\} \\
& W_{\alpha, \epsilon}^{m}=\frac{\epsilon}{\alpha}+\frac{\alpha-1}{\alpha}, M_{\alpha, \epsilon}^{m}=\frac{\epsilon}{1-\alpha}-\frac{\alpha}{1-\alpha} .
\end{aligned}
$$

Proof: See Appendix.

We now turn to the case $\alpha \in(0,1)$. In this case, $W$ and $M$ can take more than one value. More precisely, they belong, respectively, to intervals $\left[W_{\alpha, \epsilon}^{m}, W_{\alpha, \epsilon}^{M}\right]$ and $\left[M_{\alpha, \epsilon}^{m}, M_{\alpha, \epsilon}^{M}\right]$ computed by Proposition 8.

Theorem 4: A vector $c \in \mathcal{H}_{\epsilon}^{\alpha}$ iff there exits $\zeta \in[0,1]$ such that $\boldsymbol{c} \in \mathcal{W}^{\zeta W_{\alpha, \epsilon}^{M}+(1-\zeta) W_{\alpha, \epsilon}^{m}}$ and $\boldsymbol{c} \in \mathcal{M}^{\zeta M_{\alpha, \epsilon}^{m}+(1-\zeta) M_{\alpha, \epsilon}^{M}}$.

Proof: See Appendix.

Theorem 4 gives a characterization of the set $\mathcal{H}_{\epsilon}^{\alpha}$, i.e

$$
\mathcal{H}_{\epsilon}^{\alpha}=\bigcup_{\zeta \in[0,1]} \mathcal{W}^{\zeta W_{\alpha, \epsilon}^{M}+(1-\zeta) W_{\alpha, \epsilon}^{m}} \cap \mathcal{M}^{\zeta M_{\alpha, \epsilon}^{m}+(1-\zeta) M_{\alpha, \epsilon}^{M}} .
$$

Case $3(W>0$ and $M>0)$ :

Example 1: We are given two cost function coefficients $\tilde{c}_{1}=$ $(12,20,25)$ and $\tilde{c}_{2}=(10,15,25)$, with $\alpha=0.2$ and $\epsilon=$ 0.5 . Thus we have $W_{\alpha, \epsilon}^{m}=0, W_{\alpha, \epsilon}^{M}=1, M_{\alpha, \epsilon}^{m}=0.375$ and $M_{\alpha, \epsilon}^{M}=0.625$. Fig. 1 presents the set $\mathcal{H}_{0.5}^{0.2}, \mathcal{H}_{0.5}^{0.5}$ and $\mathcal{H}_{0.5}^{0.8}$. The set $\mathcal{H}_{\epsilon}^{\alpha}$ is depicted at Fig. 2 for $\alpha=0.2$. Note that the

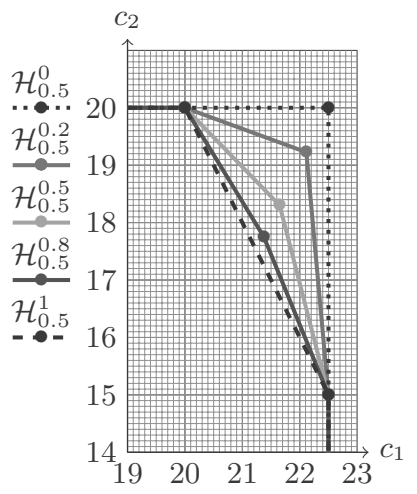

Fig. 1. Comparison of sets $\mathcal{H}_{0.5}^{\alpha}$

set $\mathcal{H}_{\epsilon}^{\alpha}$ is not convex.

We now characterize a set of possible realizations (scenarios), $\boldsymbol{c}$, of uncertain cost coefficients $\tilde{\boldsymbol{c}}$ in the adversarial problem, this allows us to show how to solve it. We need to consider three cases (in each case the set is specified by linear constraints):

Case $1\left(W=0\right.$ and $\left.M=M_{\alpha, \epsilon}^{M}\right)$ :

$$
\mathcal{C}^{1}=\left\{\boldsymbol{c}: c_{j} \leq \hat{c}_{j}+\left(1-M_{\alpha, \epsilon}^{M}\right) \Delta_{j}^{+}, j \in[n]\right\},
$$

Case $2\left(M=0\right.$ and $\left.W=W_{\alpha, \epsilon}^{M}\right)$ :

$$
\begin{aligned}
\mathcal{C}^{2}=\{\boldsymbol{c} & : \sum_{i \in[n]} u_{i}-W_{\alpha, \epsilon}^{M}=n-1, \\
& \left.c_{j} \leq \hat{c}_{j}+\left(1-u_{j}\right) \Delta_{j}^{+}, j \in[n], \boldsymbol{u} \in[0,1]^{n}\right\},
\end{aligned}
$$

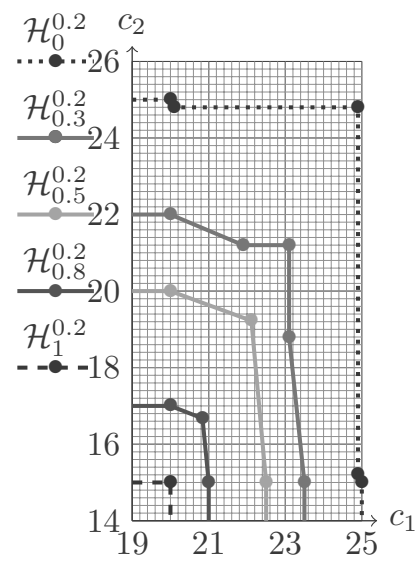

Fig. 2. Comparison of sets $\mathcal{H}_{\epsilon}^{0.2}$

$$
\begin{aligned}
\mathcal{C}^{3}=\{\boldsymbol{c} & : \sum_{i \in[n]} u_{i}-\left(\zeta W_{\alpha, \epsilon}^{M}+(1-\zeta) W_{\alpha, \epsilon}^{m}\right)=n-1, \\
& c_{j} \leq \hat{c}_{j}+\left(1-u_{j}\right) \Delta_{j}^{+}, \\
& c_{j} \leq \hat{c}_{i}+\left(1-\left(\zeta M_{\alpha, \epsilon}^{m}+(1-\zeta) M_{\alpha, \epsilon}^{M}\right)\right) \Delta_{j}^{+}, \\
& \left.j \in[n], \boldsymbol{u} \in[0,1]^{n}, \zeta \in[0,1]\right\} .
\end{aligned}
$$

Hence, depending on the maximum and minimum values of $W$ and $M$ and the value of $\alpha$ we get the following result:

Theorem 5: The ADV problem: $\max _{\boldsymbol{c} \in \mathcal{H}_{\epsilon}^{\alpha}} c^{T} \boldsymbol{x}, \alpha \in[0,1]$ is equivalent to:

$$
\begin{aligned}
& \text { - } \max _{\boldsymbol{c} \in \mathcal{C}^{1}} \boldsymbol{c}^{T} \boldsymbol{x} \text { if } \alpha=0 \text {, } \\
& \text { - } \max _{\boldsymbol{c} \in \mathcal{C}^{2}} c^{T} x \text { if } \alpha=1 \text {, } \\
& \text { - } \max _{\boldsymbol{c} \in \mathcal{C}^{3}} \boldsymbol{c}^{T} \boldsymbol{x} \text { if } W_{\alpha, \epsilon}^{m} \neq 0 \text { and } M_{\alpha, \epsilon}^{m} \neq 0 \\
& \text { - } \max \left\{\max _{\boldsymbol{c} \in \mathcal{C}^{1}} \boldsymbol{c}^{T} \boldsymbol{x}, \max _{\boldsymbol{c} \in \mathcal{C}^{2}} \boldsymbol{c}^{T} \boldsymbol{x}, \max _{\boldsymbol{c} \in \mathcal{C}^{3}} \boldsymbol{c}^{T} \boldsymbol{x}\right\} \text { if } W_{\alpha, \epsilon}^{m}=0 \text { and } \\
& M_{\alpha, \epsilon}^{m} \stackrel{\boldsymbol{c} \in \mathcal{C}}{=} 0, \\
& \text { - } \max \left\{\max _{\boldsymbol{c} \in \mathcal{C}^{1}} \boldsymbol{c}^{T} \boldsymbol{x}, \max _{\boldsymbol{c} \in \mathcal{C}^{3}} \boldsymbol{c}^{T} \boldsymbol{x}\right\} \text { if } W_{\alpha, \epsilon}^{m}=0 \text { and } M_{\alpha, \epsilon}^{m} \neq 0 \text {, } \\
& \text { - } \max \left\{\max _{\boldsymbol{c} \in \mathcal{C}^{2}} \boldsymbol{c}^{T} \boldsymbol{x}, \max _{\boldsymbol{c} \in \mathcal{C}^{3}} \boldsymbol{c}^{T} \boldsymbol{x}\right\} \text { if } W_{\alpha, \epsilon}^{m} \neq 0 \text { and } M_{\alpha, \epsilon}^{m}=0 \text {. }
\end{aligned}
$$

\section{Resolution of Fuzzy Linear Problem with COPULA $H_{\alpha}$}

Before we propose an algorithm for solving the problem (13), we will focus on the problem (13) for a fixed $\epsilon$. Thus it boils down to checking if constraint $\max _{\boldsymbol{c} \in \mathcal{H}_{\epsilon}^{\alpha}} \boldsymbol{c}^{T} \boldsymbol{x} \leq g$ is satisfied. For the sake of brevity, we only consider the case when $W_{\alpha, \epsilon}^{m} \neq 0$ and $M_{\alpha, \epsilon}^{m} \neq 0$, the other cases can handled in a similar manner. Consider the following mathematical 
programming problem:

$$
\begin{array}{cl}
\min & h^{\epsilon} \\
\text { s.t. } & \max _{\boldsymbol{c} \in \mathcal{C}^{1}} \boldsymbol{c}^{T} \boldsymbol{x} \leq h^{\epsilon}, \\
& \max _{\boldsymbol{c} \in \mathcal{C}^{2}} \boldsymbol{c}^{T} \boldsymbol{x} \leq h^{\epsilon}, \\
& \max _{\boldsymbol{c} \in \mathcal{C}^{3}} \boldsymbol{c}^{T} \boldsymbol{x} \leq h^{\epsilon}, \\
& \boldsymbol{a}_{i}^{T} \boldsymbol{x} \geq b_{i} \\
& \boldsymbol{x} \geq \mathbf{0} .
\end{array}
$$

From theorem 5 it follows that $\max _{\boldsymbol{c} \in \mathcal{H}_{\epsilon}^{\alpha}} \boldsymbol{c}^{T} \boldsymbol{x} \leq g$ is satisfied if an optimal value of $h^{\epsilon}$, in problem (15)-(18) for a fixed $x$, is less than or equal to $g$. Furthermore it is easy to give a linear programming formulation of the problem (15)-(18) by dualizing the left hand sides of the constraints (16), (17) and (18).

We are now ready to propose an algorithm for solving problem (13), which is based on the standard binary search in $[0,1]$ (the interval of possible values of $\epsilon$ ) due to the fact that $h^{\epsilon}$ is nonincreasing function of $\epsilon$. We call it the binary search based algorithm. In order to find an optimal $\left(x^{*}, \epsilon^{*}\right)$ with a given error tolerance $\xi>0$, we seek at each iteration, for a fixed $\epsilon$, a feasible solution $x$ for which $h^{\epsilon} \leq g$ is satisfied. This which boils down to solving the linear programing formulation of the problem (15)-(20). The running time of the above algorithm is $O\left(I(T) \log \xi^{-1}\right)$ time, where $I(|T|)$ is the time required for solving the linear program corresponding to (15)(20).

\section{ILlustrative EXAMPLE}

Consider the following linear problem:

$$
\begin{array}{ll}
\max & \mathrm{N}\left(\tilde{\boldsymbol{c}}^{T} \boldsymbol{x} \leq g\right) \\
\text { s.t. } & \sum_{j \in[3]} x_{j}=2, \\
& x_{j} \in[0.5,1], \quad j \in[3],
\end{array}
$$

where $\tilde{c}_{1}=(18,20,20), \tilde{c}_{2}=(10,15,20), \tilde{c}_{3}=(5,10,22)$ and $g=34$. To solve the problem we used the binary search based algorithm. Let us first focus on two extremes cases, i.e. for $\alpha=0$ an optimal solution is $x^{(1)}=[0.5,0.5,1]$ with the necessity degree $\mathrm{N}\left(\widetilde{c}^{T} \boldsymbol{x}^{(1)} \leq g\right) \approx 0.52$ and for $\alpha=1$ an optimal solution is $x^{(2)}=[0.5,1,0.5]$ with the necessity degree $\mathrm{N}\left(\widetilde{c}^{T} x^{(2)} \leq g\right) \approx 0.834$. Fig. 3 shows that dependencies influence strongly the possibility distribution of cost. Moreover, it can be seen that the necessity degree depends on the knowledge on the dependencies, positive or negative. If the costs coefficients increase simultaneously the certainty to have a solution with cost lower than 34 is low. On the contrary, when one cost coefficient increases and the others decrease the necessity degree to have a solution with cost lower than 34 is high.

Let us now analyze the impact of the strength $\alpha \in[0,1]$ of the positive/negative dependencies. Fig. 4 shows the evolution of the necessity degree as function of $\alpha$. As expected the necessity degree increases when $\alpha$ increases. So adding the

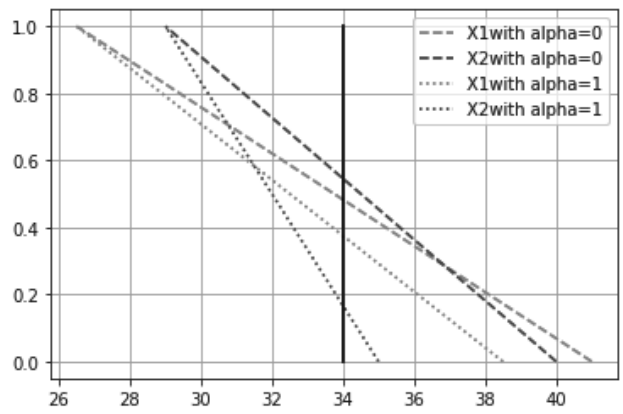

Fig. 3. Upper side of possibility distribution for positives/negatives dependencies

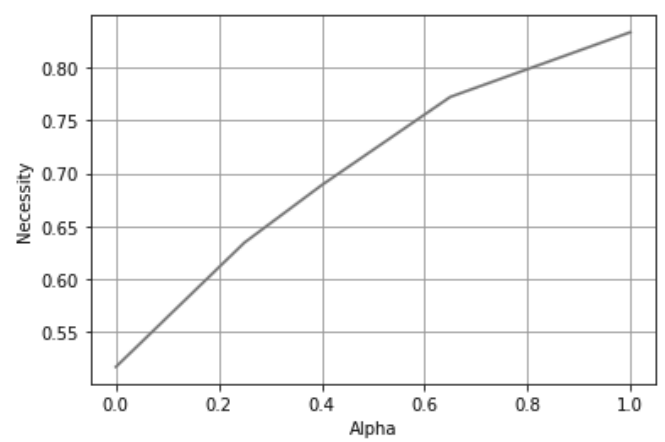

Fig. 4. Necessity degree

knowledge on the dependencies allows us a better estimation of the necessity degree.

Fig. 5 shows the upper bound of the possibilities distribution of an optimal solution for different values of $\alpha$. Fig. 6 below shows an optimal solution for different values of $\alpha$. The dependencies influence the preference between $x_{3}$ and $x_{2}$ when $\alpha$ increases. The value of $x_{3}$ passes smoothly from 1 to 0.5 when $x_{3}$ passes from 0.5 to 1 .

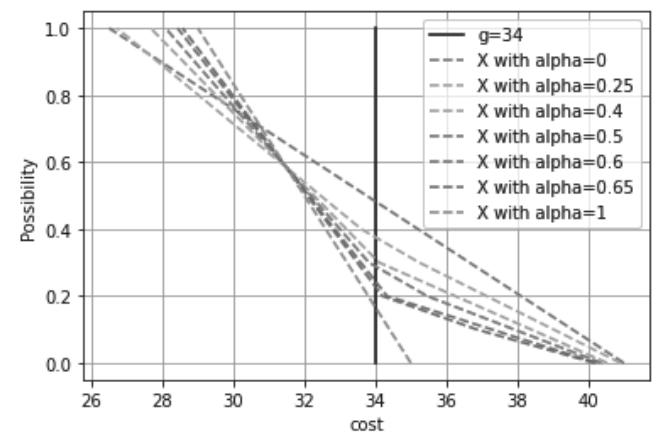

Fig. 5. Upper side of possibility distribution of cost for an optimal solution

\section{CONCLUSION}

In this paper we have proposed a new model to take into account the knowledge on uncertain cost function coefficient dependencies by a generalization of copula functions to the 


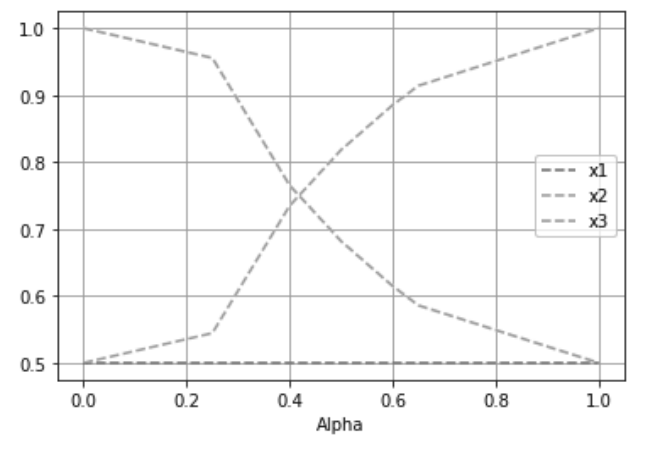

Fig. 6. Evolution of an optimal solution

possibilistic case. It has turned out that taking into account these dependencies in a linear programming problem with uncertain cost coefficients in the objective does not increase the computational complexity, i.e. a resulting problem remains a linear programming one. Moreover, we have shown that taking into account dependencies allows better estimation of the necessity measure and has influence on choosing an optimal solution.

\section{REFERENCES}

[1] D. Dubois and H. Prade, Possibility theory: an approach to computerized processing of uncertainty. New York: Plenum Press, 1988.

[2] D. Dubois, "Possibility theory and statistical reasoning," Computational statistics \& data analysis, vol. 51, no. 1, pp. 47-69, 2006.

[3] D. Dubois, H. Fargier, and P. Fortemps, "Fuzzy scheduling: Modelling flexible constraints vs. coping with incomplete knowledge," European Journal of Operational Research, vol. 147, no. 2, pp. 231-252, 2003.

[4] R. Guillaume, A. Kasperski, and P. Zieliński, "Robust possibilistic production planning under budgeted demand uncertainty," in 2020 IEEE International Conference on Fuzzy Systems (FUZZ-IEEE). IEEE, 2020, pp. $1-8$.

[5] E. Nikolova, "Approximation algorithms for offline risk-averse combinatorial optimization," in Proceedings of APPROX'10, 2010.

[6] B. Liu, "Fuzzy random chance-constrained programming," IEEE Transactions on Fuzzy systems, vol. 9, no. 5, pp. 713-720, 2001.

[7] H. Fargier and C. Thierry, "The use of possibilistic decision theory in manufacturing, planning and control: recent results in fuzzy master production scheduling," Studies in fuzziness and soft computing. SpinglerVerlag, vol. 36, pp. 45-59, 2000.

[8] R. Guillaume, B. Grabot, and C. Thierry, "Management of the risk of backorders in a mto-ato/mts context under imperfect requirements," Applied Mathematical Modelling, vol. 37, no. 16-17, pp. 8060-8078, 2013.

[9] P. Mikusiński, H. Sherwood, and M. Taylor, "Probabilistic interpretations of copulas and their convex sums," in Advances in probability distributions with given marginals. Springer, 1991, pp. 95-112.

[10] R. B. Nelsen, An introduction to copulas. Springer Science \& Business Media, 2007.

[11] F. Durante and C. Sempi, "Copula theory: An introduction," in Copula Theory and its Applications. Springer, 2010, pp. 3-31.

\section{APPENDIX}

Proof of Proposition 1: Write $\Omega_{\lambda}^{C}=\{c$ : $\left.C\left(\Pi_{1}\left(c_{1}\right), \ldots, \Pi_{n}\left(c_{n}\right)\right) \geq \lambda\right\}$ and $\Omega_{\lambda}^{\breve{C}}=\{\boldsymbol{c}:$ $\left.\breve{C}\left(\bar{\Pi}_{1}\left(c_{1}\right), \ldots, \bar{\Pi}_{n}\left(c_{n}\right)\right) \geq \bar{\lambda}\right\}$. From the definition of the copula (see Sect. II) we get $\Omega_{\lambda_{1}}^{C} \subseteq \Omega_{\lambda_{2}}^{C}$ for every $\lambda_{1}, \lambda_{2} \in[0,1]$ such that $\lambda_{1} \geq \lambda_{2}$ (resp. $\Omega_{\lambda_{1}}^{C} \subseteq \Omega_{\lambda_{2}}^{C}$ for every $\lambda_{1}, \lambda_{2} \in[0,1]$ such that $\left.\lambda_{1} \geq \lambda_{2}\right)$. Hence $\left(\Omega_{\lambda_{1}}^{C} \cap \Omega_{\lambda_{1}}^{\breve{C}}\right) \subseteq\left(\Omega_{\lambda_{2}}^{C} \cap \Omega_{\lambda_{2}}^{\breve{C}}\right)$ for every $\lambda_{1}, \lambda_{2} \in[0,1]$ such that $\lambda_{1} \geq \lambda_{2}$.
Proof of Proposition 2: Let be $c^{*}$ a vector such that $\pi_{\tilde{c}_{j}}\left(c_{j}^{*}\right)=1, j \in[n]$. Such a vector exists since univariate possibility distributions are normalized. From the definition of the copula (see Sect. II), we obtain $C\left(\Pi_{1}\left(c_{1}^{*}\right), \ldots, \Pi_{n}\left(c_{n}^{*}\right)\right)=$ 1 and from the continuity of $\pi_{\tilde{c}_{j}}$ we have $\bar{\Pi}_{j}\left(c_{j}^{*}\right)=1$ for every $j \in[n]$. Again the definition of the copula function yields $\breve{C}\left(\bar{\Pi}_{1}\left(c_{1}^{*}\right), \ldots, \bar{\Pi}_{n}\left(c_{n}^{*}\right)\right)=1$. So the $\lambda$-cut for $\lambda=1$ have at least one $c^{*}$.

Proof of Proposition 3: Our proof starts with the observation that for each $\pi_{\tilde{c}} \in \mathcal{P}_{(C, \breve{C})}$ we have the equality: $\sup _{\boldsymbol{c}: \boldsymbol{c}^{T} \boldsymbol{x} \leq g} \pi(\boldsymbol{c})=\sup _{\boldsymbol{c}: \boldsymbol{c}^{T} \boldsymbol{x} \leq g} \Pi(\tilde{\boldsymbol{c}}=\boldsymbol{c})$. Furthermore since $\boldsymbol{x} \geq \mathbf{0}$ and $\boldsymbol{c}^{T} \boldsymbol{x} \leq g$, the constraint $\boldsymbol{c}^{T} \boldsymbol{x} \leq g$ is satisfied for every $\boldsymbol{c}^{\prime}$ such that $\boldsymbol{c}^{\prime} \leq \boldsymbol{c}$. Hence $\pi_{\tilde{c}} \in \mathcal{P}_{(C, \breve{C})}$ $\sup _{\boldsymbol{c}: \boldsymbol{c}^{T} \boldsymbol{x} \leq g} \Pi(\tilde{\boldsymbol{c}}=\boldsymbol{c})=\sup _{\boldsymbol{c}: \boldsymbol{c}^{T} \boldsymbol{x} \leq g} \Pi(\tilde{\boldsymbol{c}} \leq \boldsymbol{c})$. From (9) it follows that for each $\pi_{\tilde{\boldsymbol{c}}} \in \mathcal{P}_{(C, \breve{C})} \Pi\left(\widetilde{\boldsymbol{c}}^{T} \boldsymbol{x} \leq g\right)=$ $\sup _{\boldsymbol{c}: \boldsymbol{c}^{T} \boldsymbol{x} \leq g} C\left(\Pi_{1}\left(c_{1}\right), \ldots, \Pi_{n}\left(c_{n}\right)\right)$.

Proof of Proposition 4: A proof is similar to the one of Proposition 3. It is sufficient to use the possibility and necessity measure relationship together with (10).

Proof of Proposition 5: Since $W(\boldsymbol{u})$ and $M(\boldsymbol{u})$ are copulas for $n=2$, their convex combination is a copula as well (see [9], [11]).

Proof of Proposition 6: From [10, Theorem 2.10.13] it follows that there exists a copula $C \in \mathcal{C}_{n}, n \leq 3$, (which depends on $\boldsymbol{u}$ ) such that $C(\boldsymbol{u})=W(\boldsymbol{u})$ for any $\boldsymbol{u} \in[0,1]^{n}$. $M(\boldsymbol{u})$ is a copula. Hence their convex combination is a copula as well (see [9], [11]).

Proof of Proposition 7: 1 - $^{-}$ $\sup _{\boldsymbol{c}: \boldsymbol{c}^{T} \boldsymbol{x} \geq g} H_{\alpha}\left(\bar{\Pi}_{1}\left(c_{1}\right), \ldots, \bar{\Pi}_{n}\left(c_{n}\right)\right)>1-\epsilon \Longleftrightarrow$ $\sup _{\boldsymbol{c}: \boldsymbol{c}^{T} \boldsymbol{x}>g} H_{\alpha}\left(\bar{\Pi}_{1}\left(c_{1}\right), \ldots, \bar{\Pi}_{n}\left(c_{n}\right)\right)<\epsilon \Longleftrightarrow \nexists \boldsymbol{c}: \boldsymbol{c}^{T} \boldsymbol{x}>g$ with $H_{\alpha}\left(\bar{\Pi}_{1}\left(c_{1}\right), \ldots, \bar{\Pi}_{n}\left(c_{n}\right)\right) \geq \epsilon$.

Proof of Proposition 8: The set of values of $\mathrm{M}$ and $\mathrm{W}$ can be represented by a set of linear constraints:

$$
\begin{aligned}
& \epsilon=\alpha W+(1-\alpha) M, \\
& W, M \in[0,1] .
\end{aligned}
$$

We can see that this system of equation is never empty ( $W=$ $M$ is always a solution). Moreover the maximum value of $W$ for a given $\alpha, \epsilon$ noted $W_{\alpha, \epsilon}^{M}$ is obtain for the minimum value of $M$ noted $M_{\alpha, \epsilon}^{m}$ and vice versa.

Proof of Theorem 4: We start with some observations, namely for each $\epsilon_{1}, \epsilon_{2} \in[0,1]$ such that $\epsilon_{1}>\epsilon_{2}$ we have:

- $W_{\alpha, \epsilon_{1}}^{M} \geq W_{\alpha, \epsilon_{2}}^{M}$,

- $W^{\alpha, \epsilon_{1}} \geq W^{\alpha, \epsilon_{2}}$,

- $M_{\alpha, \epsilon_{1}}^{M, \epsilon_{1}} \geq M_{\alpha, \epsilon_{2}}^{M}$,

- $M_{\alpha, \epsilon_{1}}^{m} \geq M_{\alpha, \epsilon_{2}}^{m}$,

- $\mathcal{M}^{\epsilon_{1}} \subseteq \mathcal{M}^{\epsilon_{2}}$

- $\mathcal{W}^{\epsilon_{1}} \subseteq \mathcal{W}^{\epsilon_{2}}$.

Accordingly, we have $\mathcal{H}_{\epsilon_{1}}^{\alpha} \subseteq \mathcal{H}_{\epsilon_{2}}^{\alpha}$ for every $\epsilon_{1}, \epsilon_{2} \in[0,1]$ such that $\epsilon_{1}>\epsilon_{2}$. Hence the set $\mathcal{H}_{\epsilon}^{\alpha}$ depends only on the sets $\mathcal{W}^{\epsilon_{W}}$ and $\mathcal{M}^{\epsilon_{M}}$ such that $\epsilon_{W}=\zeta W_{\alpha, \epsilon}^{M}+(1-\zeta) W_{\alpha, \epsilon}^{m}$ and $\epsilon_{M}=\zeta M_{\alpha, \epsilon}^{m}+(1-\zeta) M_{\alpha, \epsilon}^{M}$ for every $\zeta \in[0,1]$. 